Check for updates

Cite this: RSC Adv., 2018, 8, 20836

\section{Micropatterned immobilization of membrane- mimicking polymer and peptides for regulation of cell behaviors in vitro $\uparrow$}

\author{
Wenyong Ma, (D) Luying Liu, Huiqing Chen, Yuancong Zhao, (D) * Ping Yang* \\ and Nan Huang
}

To regulate the behaviors and functions of endothelial cells (ECs) on the biomaterials on titanium (Ti), a biomimetic micropattern (ridge/groove: 25/25 $\mu \mathrm{m}$ ) of polymer of 2-methacryloyloxyethyl phosphorylcholine (polyMPC) and Gly-Arg-Glu-Asp-Val-Tyr (GREDVY) was fabricated. PMMPC (monomer contain MPC and methacrylic acid (MA)) containing carboxyl groups was chosen, and PMMPC was cross-linked with hexamethylene diamine through condensation reaction of amino and carboxyl. Simultaneously, the carboxyl groups of cross-linked PMMPC (PMMPC-HD) can react with amino groups of polydopamine which can adhered on many materials firmly. GREDVY was immobilized on polydopamine but not on PMMPC-HD because amino and carboxyl groups can react with catechol and amino groups of polydopamine. IR and ${ }^{1} \mathrm{H}$ NMR demonstrated that PMMPC-HD was successfully synthesized. And the QCM-D (quartz crystal microbalance with dissipation) and IR approved that PMMPC-HD and GREDVY can be immobilized on polydopamine (PDA). Platelet adhesion and whole blood adhesion on micropattern modificated with PMMPC and GREDVY (Ti-PDA-M/R(P)) showed better hemocompatibility than other samples. Endothelial cells were regulated in the direction of micropattern showing elongated ECs were closer to a healthy, athero-protective phenotype than ECs cultured in vitro without micropattern. $\mathrm{NO}$ and $\mathrm{PGl}_{2}$ release were upregulated. Simultaneously the number of SMCs on Ti-PDA-M/R(P) was the smaller that of other samples, which demonstrated that the Ti-PDA-M/R(P) had property of inhibiting SMCs proliferation to a certain extent.
Received 26th March 2018 Accepted 28th May 2018

DOI: $10.1039 / c 8 \mathrm{ra02607f}$

rsc.li/rsc-advances part in biocompatibility. To further regulate ECs behaviors, many micropatterns on surfaces of biomaterials were fabricated. For example, Kuribayashi ${ }^{7}$ fabricated micropatterns of the MPC polymer of different sizes on glass and seeded ECs on them. The data demonstrated the ECs were aligned in the direction of micropattern at the single-cell level. Huang ${ }^{8}$ used parallel-aligned micropatterned substrate made of PDMS (polydimethylsiloxane) covered with collagen type I to evaluate the effects of spatial patterning on the phenotype of ECs, and they demonstrated that micropatterned biomaterials could effectively guide ECs organization along the direction of the micropatterned channels and promote an athero-resistant ECs phenotype by reducing endothelial adhesiveness for monocytes and platelets. To further study patterned ECs in potential application in cardiovascular stents, titanium was chosen as a substrate materials, and micropatterned immobilization of cytophilic and cytophobic materials on them. It is well accepted that titanium (Ti) is a promising material can be used in many biomedical fields, such as cardiovascular implants (prosthetic heart valves, protective cases for pacemakers, artificial hearts and circulatory devices), hard tissue replacements and so on., ${ }^{9,10}$ Nickel-titanium alloy stents are often used in treatment of
Key Laboratory of Advanced Technology of Materials, Ministry of Education, School of Material Science and Technology of Southwest Jiaotong University, Chengdu 610031, PR China. E-mail: Zhaoyc7320@163.com; Yangping8@263.net; Fax: +86-28-8760625; Tel: $+86-28-87634148$

$\dagger$ Electronic supplementary information (ESI) available. See DOI: $10.1039 / \mathrm{c} 8 \mathrm{ra} 02607 \mathrm{f}$ 
cardiovascular disease and are usually coated with a thin carbon film to enhance blood compatibility. ${ }^{11}$

Monomer of 2-methacryloyloxyethyl phosphorylcholine was applied to synthesize polymer for better hemocompatibility. ${ }^{\mathbf{1 2 - 1 5}}$ Inspired by the anticoagulation property of outer membrane, 2methacryloyloxyethyl phosphorylcholine, which is a methacrylate monomer and bears a polar group same as in the natural phospholipid molecules, was synthesized. It demonstrated that polyMPC has the ability of protein non-specific resistance and better antioxidant and hydration properties than PEG which was widely applied in biomedical fields. ${ }^{12,16-20}$ For example, Feng $^{18}$ modified the surface of silicon wafer with polyMPC via surface initiated atom transfer polymerization and showed that suppression of protein adsorption on the polyMPC grafted surfaces was not strongly dependent on protein size or charge. PolyMPC has good hemocompatibility, but is non-specific resistance adhesion of endothelial cell. ${ }^{7,21,22}$ To better adhere endothelial cells, peptides Arg-Glu-Asp-Val (REDV) ${ }^{23-26}$ derived from fibronectin has the ability to adhere EC specifically compared to smooth muscle cell, was widely applied in the field of surface modification of biomaterials. It is because REDV ligand can specifically bind with integrin $\alpha_{4} \beta_{1}$ in the membrane of endothelial cells, but integrin $\alpha_{4} \beta_{1}$ was not in vascular smooth cells and platelets. ${ }^{27-30} \mathrm{REDV}^{23}$ was grafted onto the side chain of polyMPC and the results of surface modified with the polymer demonstrated that the competitive adhesion and the growth of ECs over SMCs could be enhanced by the synergic action of the nonspecific resistance to $\mathrm{PC}$ and the specific recognition of the REDV peptide. Plouffe ${ }^{31}$ demonstrated that the adhesion of ECs in REDV-coated devices becomes significantly greater than that of the non targeted cells with decreasing shear stress, and the adhesion of ECs is shown to be independent whether ECs flow through the devices as suspensions of only one cell type or as a heterogeneous suspension.

To immobilize PMMPC on Ti, polydopamine was deposited on the Ti substrate bearing amino and quinone group. Hexamethylene diamine can react with carboxyl of PMMPC, where PMMPC bearing carboxyl group was cross-linked and named PMMPC-HD. The amino groups of polydopamine could react with carboxyl groups of PMMPC-HD through condensation reaction, therefore PMMPC-HD can be immobilized on Ti deposited with polydopamine covalently. Peptide GREDVY was used to maintain the integrity and functionality of REDV, and the peptide bearing amino and carboxyl groups could be immobilized on polydopamine coating.

To make a further study morphology and functions of ECs on micropattern, the micropattern was fabricated with PMMPC-HD utilizing microtransfer molding method, and REDV which contains amino and carboxyl group was covalently immobilized on polydopamine via condensation reaction, Michael addition and Schiff base reaction, where polyMPC was cytophobic and the REDV peptides were cytophilic. ECs were adhered on the stripe of REDV, and orientation of ECs was in the direction of striped micropattern. The functions of ECs, nitric oxide (NO) and $\mathrm{PGI}_{2}$, were evaluated, and the data showed that functions of ECs on the biomimetic micropattern (Ti-PDA-M/R(P)) were much closer to the natural ECs than that of unmodified Ti sample.

\section{Materials and methods}

\subsection{Materials}

Titanium (Ti) was obtained from New Material Co., Ltd (Xi'an, China). Dopamine was purchased from Sigma-Aldrich (USA). Hexamethylene diamine (HD) was bought from Jinshan Chemical Reagent (Chengdu, China). 2-Methacryloyloxyethyl phosphorylcholine (MPC, 96\%) was supplied by the Joy-Nature Technology Institute (China) and used without further purification. PDMS prepolymer and catalysts (Sylgard 184) were purchased from Dow Corning (Midland, MI, USA). 1-(3Dimethylaminopropyl)-3-ethylcarbodiimide hydrochloride (EDC) and $N$-hydroxysuccinimide (NHS) were from SigmaAldrich. GREDVY (Shanghai Science Peptide Biological Technology Co., ltd), DMEM (Dulbecco's modified eagle medium), pancreatin and FBS (fetal bovine serum) were purchased from GIBCO (USA).

\subsection{Polydopamine deposited onto titanium surface}

Titanium was cut into wafers of $10 \mathrm{~mm}$ in diameter, then polished and ultrasonic cleaned with ethanol, acetone and deionized water. The titanium plates were immersed into dopamine solution which was prepared by way of dissolving dopamine hydrochloride $\left(2 \mathrm{mg} \mathrm{mL} \mathrm{mL}^{-1}\right)$ in Tris-base buffer $(10 \mathrm{mM}, \mathrm{pH}=$ 8.5). After $12 \mathrm{~h}$ at room temperature, polydopamine coated titanium was ultrasonically washed with distilled water $(10 \mathrm{~min}$ $\times 3$ times). The above process was repeated for 4 times, and the surface of titanium wafer covered with polydopamine was obtained and named Ti-PDA.

\subsection{Preparation of cross-linked PMMPC-HD copolymer for micropattern immobilization}

The polymer of PMMPC (mol\%, MPC/MA = 7/3) was synthesized according to the reported method. ${ }^{32}$ The relative molecular mass of PMMPC was determined by Gel Permeation Chromatography (GPC), the $M_{\mathrm{w}}$ was $170 \mathrm{kDa}\left(M_{\mathrm{w}} / M_{\mathrm{n}}=1.34\right)$. To cross-link PMMPC with hexamethylene diamine, the PMMPC (100 mg) was put into flask with $95 \mathrm{~mL}$ deionized water and $5 \mathrm{~mL}$ ethanol, and then $100 \mathrm{mg}$ of EDC and $25 \mathrm{mg}$ of NHS were added, for activation carboxyl. After being stirred for $15 \mathrm{~min}$, $10 \mathrm{mg}$ of hexamethylene diamine (maintaining the molar ratio of $-\mathrm{NH}_{2}$ to $-\mathrm{COOH}$ was $1: 1$ ) was added, too. After being stirred for $3 \mathrm{~h}$ at room temperature under Ar gas, the solution of mixed cross-linked PMMPC-HD was concentrated to about $10 \mathrm{mg}$ $\mathrm{mL}^{-1}$ which was used to fabricate micropattern of PMMPC-HD. To characterize the crossing-linked PMMPC-HD, the mixture was transferred into dialysis tubing (molecular weight cut off: 8000 ) and stirred in deionized water for $48 \mathrm{~h}$ to remove the small molecules. The cross-linked polymer called PMMPC-HD was obtained through freeze-drying. The reaction was shown in Fig. 1(d).

\subsection{Fabrication of PDMS stamps}

PDMS stamps were fabricated by casting a $10: 1(\mathrm{v} / \mathrm{v})$ mixture of monomers and curing agents against a complementary relief 
(a)

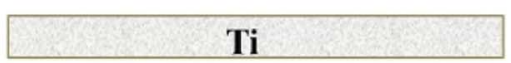
Ti

Tris-base buffer (pH 8.5)
11111111111111111111111

Ti

Ti-PDA-REDV

(b)
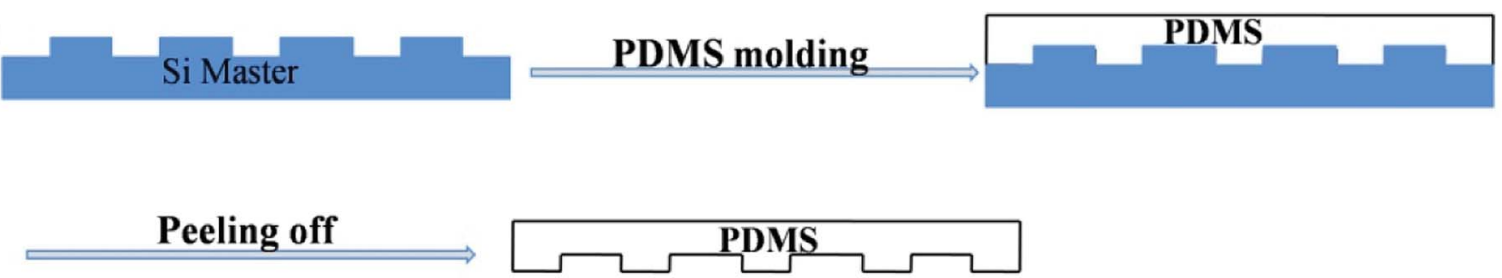

(c)
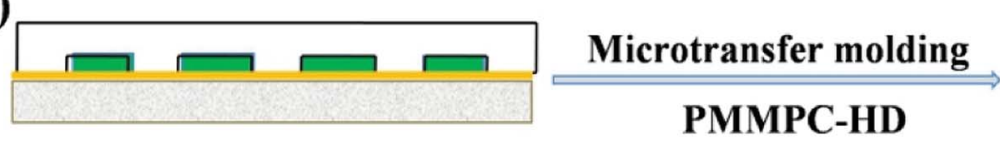

PMMPC-HD

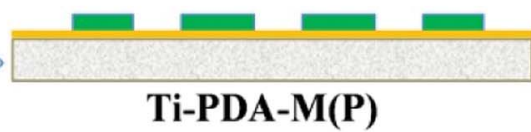

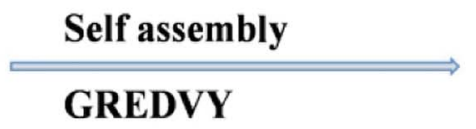

Polydopamine



Ti-PDA-M/R(P)

\section{Ti-PDA}

Ti 


\subsection{Micropatterned immobilization of PMMPC-HD and peptide REDV}

PDMS stamps were treated in an oxygen plasma, an inductively coupled plasma excited by an external copper band electrode at

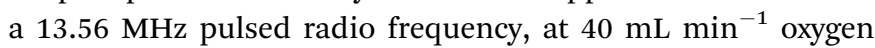
flow rate, under $100 \mathrm{~W}$ radio frequency power for $10 \mathrm{~min}$. A cotton swab was wetted with a solution of mixed cross-linked PMMPC-HD and dragged across the surface of the PDMS stamp to wet the molded PDMS stamp. The stamp was then dried with a stream of nitrogen and brought into contact with the samples of Ti-PDA for about $30 \mathrm{~min}$, after which the stamp was peeled away from the substrate. Finally, to remove the EDC/ NHS and hexamethylene diamine which not reacted, the micropatterned sample was immersed in deionized water for $24 \mathrm{~h}$, and the micropattern of PMMPC-HD was obtained and named Ti-PDA-M(P). In other words, the band without PMMPCHD was PDA surface. Subsequently, Ti-PDA-M(P) were immersed in a solution of GREDVY at $37{ }^{\circ} \mathrm{C}$ for $2 \mathrm{~h}$ and then rinsed with PBS to remove the nonattached GREDVY. Here in PBS the concentration of GREDVY was $300 \mu \mathrm{g} \mathrm{mL}{ }^{-1}$, and the striped pattern was named Ti-PDA-M/R(P). The whole process was shown in Fig. 1(a-c).

In an attempt to confirm that PMMPC-HD and GREDVY could adhere onto the surface of polydopamine covalently, a quartz crystal microbalance (QCM) sensor with dissipation monitoring (QCM-D, Q-Sense, Gothenburg, Sweden) was used to detect the amount of it and its stability. The QCM is widely used to measure the change in a mass $(\Delta m)$ of materials/ molecules attached to the surface of the QCM sensor via changes in the resonant frequency $(\Delta f)$. The mass of the adhered layer can be calculated using the Sauerbrey equation:

$$
\Delta m=-C \times \Delta f n / n
$$

where $C=17.7 \mathrm{ng} \mathrm{cm}{ }^{-2} \mathrm{~Hz}^{-1}, n$ is the overtone number $(n=1$, $3,5,7$.$) , and f n$ is the frequency of the overtone. ${ }^{34}$ The QCM sensor deposited with polydopamine (Au-PDA) for $4 \mathrm{~h}$ was exposed to the EDC/NHS solution until a stable baseline of the QCM signals was obtained. The QCM cell was then filled with $2.0 \mathrm{mg} \mathrm{mL} \mathrm{m}^{-1}$ of mixed cross-linked PMMPC-HD. After the PMMPC-HD solution was retained for $20 \mathrm{~min}$ in the QCM cell, deionized water solution was added to replace the PMMPC-HD solution and wash away the weakly adsorbed PMMPC-HD from the surface. The QCM signals were monitored throughout the procedure. All the measurements were performed at $25{ }^{\circ} \mathrm{C}$ and repeated at least three times. Similarly, the GREDVY in PBS $(300$ $\mu \mathrm{g} \mathrm{mL}^{-1}$ ) was used to detect the adhesion mass in QCM, PBS solution was used to clean the surface of QCM sensor and GREDVY which was not immobilized firmly. The QCM test was repeated twice.

\subsection{Characterization of PMMPC-HD}

PMMPC-HD was determined by FTIR (NICOLET 5700, USA) with the mode of transmission. The dried polymer was grinded uniformly with solid $\mathrm{KBr}$, then the mixture was pressed into a thin film so as to detect the functional groups.
To demonstrate that the PMMPC can be crosslinked with HD, ${ }^{1} \mathrm{H}$ NMR (Varian INOVA-400) spectra of PMMPC-HD was characterized. The polymer PMMPC-HD with low degree of cross-linking (reaction time: $20 \mathrm{~min}$ white translucent flaky solid) was dissolved in $\mathrm{D}_{2} \mathrm{O}$, then was put into NMR tube for the purpose of detecting the hydrogen atom in different chemical environment (chemical shift $(\delta)$ ), using TMS as the internal standard.

\subsection{Surface characterization}

The morphologies of bare $\mathrm{Ti}$, modified $\mathrm{Ti}$ and micropatterned samples were observed by scanning electron microscopy (SEM, JSM-7001F, Japan), and the surface chemical composition was examined using Fourier transform infrared spectrometer (FTIR, NICOLET 5700, USA) with the mode of infrared grazing. Static water contact angles were measured using a DSA100 Drop Shape Analyzer at room temperature. At least 6 contact angles in different areas were measured and averaged. The samples were washed with ethanol and dried in Ar stream immediately before the measurement. The thickness of PMMPC-HD was measured using AFM (AFM, Nihon Veeco, Tokyo, Japan).

\subsection{Platelets adhesion and whole blood adhesion}

Platelet adhesion test was carried out to evaluate the adhesion, distribution, behavior and activation of the platelets in vitro. First, fresh whole blood supplied by the center of blood station of Chengdu containing anticoagulant with $0.109 \mathrm{M}$ solution of sodium citrate at a dilution ratio of $9: 1$ (blood/sodium citrate solution) was centrifuged at $1500 \mathrm{rpm}$ for $15 \mathrm{~min}$ to separate the blood corpuscles and get platelet rich plasma (PRP). Second, $300 \mu \mathrm{L}$ PRP was added on the samples and incubated at $37{ }^{\circ} \mathrm{C}$ for $15 \mathrm{~min}$. Subsequently, the samples were rinsed three times with PBS and fixed with $2.5 \%$ glutaraldehyde for $4 \mathrm{~h}$. Finally, the samples stained with rhodamine 123 were observed through fluorescence microscope.

Whole blood adhesion test was prepared as follows: $300 \mu \mathrm{L}$ fresh whole blood containing anticoagulant was added on the samples and incubated at $37^{\circ} \mathrm{C}$ for $30 \mathrm{~min}$. Then, the samples were rinsed three times with PBS and fixed with $2.5 \%$ glutaraldehyde for $4 \mathrm{~h}$. Finally, the samples were dehydrated with 50, 75, 90 and $100 \%(\mathrm{v} / \mathrm{v})$ ethanol water solution in turn every for 15 min and observed by way of SEM.

The study complied with all Institutional and National Guidelines, as per China Association for Ethical Studies (CAES) Statement: Ethical Conduct for Research Involving Humans, the protocol was approved by Southwest Jiaotong University Human Research Ethics Board, and all participants provided written informed consent.

\subsection{ECs culture}

ECs derived from human umbilical vein were isolated and cultured by using the following method. A human umbilical cord was cannulated and washed thoroughly with PBS to remove the blood inside the lumen. And then $0.1 \%$ type II collagenase, the human umbilical cord was incubated (Invitrogen Corporation) in medium 199 at $37{ }^{\circ} \mathrm{C}$ for $15 \mathrm{~min}$. The 
detached cells were washed in serum-free medium and collected in complete M199 containing 15\% fetal bovine serum (FBS, Hyclone Company). The suspended cells were then seeded in a single-used culture flask and incubated at $37{ }^{\circ} \mathrm{C}$ in a humidified atmosphere with $95 \%$ air and $5 \% \mathrm{CO}_{2}$. Harvested primary ECs could be used to evaluate the proliferation behavior on samples.

Ti, Ti-PDA, and Ti-PDA-M(P) samples were sterilized in a sealed box exposed to the UV light for $1 \mathrm{~h}$, Ti-PDA-REDV, and Ti-PDA-M/R(P) samples was fabricated using filtered solution of GREDVY and sterilized Ti-PDA and Ti-PDA-M(P). All the sterilized samples were placed in a 24-well culture plate, and $1 \mathrm{~mL}$ of EC suspension was added. The culture time was 1 day (1D) and 3 days (3D). The concentration of EC used for seeding was $4 \times$ $10^{4}$ cells per mL. To study the morphology of ECs on samples, the ECs were stained with rhodamine 123 (a fluorescent molecule), the procedure was as follows: after the cells were fixed with $2.5 \%$ glutaraldehyde for $4 \mathrm{~h}$. The samples were washed with PBS for three times and dried in air, sample surfaces were covered with rhodamine $123\left(10 \mu \mathrm{g} \mathrm{mL}^{-1}\right)$ for $15 \mathrm{~min}$. Finally, the samples were rinsed with PBS for three times and observed through a fluorescence microscope (OLYMPUS-IX 51, Japan).

\subsection{NO and $\mathrm{PGI}_{2}$ release of ECs}

For NO and $\mathrm{PGI}_{2}$ detection, high density of ECs $\left(5 \times 10^{5}\right.$ cells per $\mathrm{mL}$ ) were seeded onto each sample to ensure the formation of a confluent monolayer. After adhesion for 1 day, to 3 days, all the samples were taken out, and a $200 \mu \mathrm{L}$ culture medium was collected respectively. All the collected culture medium was stored at $-20{ }^{\circ} \mathrm{C}$ until use. Nitric oxide release was indicated by the content of nitrite, which is a stable degradation product of NO and was detected by using Griess reagent (Sigma). In brief, the collected culture media was centrifuged to remove any particles. The supernatant $(100 \mu \mathrm{L})$ was transferred into a new 96-well plate, and then an equal volume of Griess reagent was added. The mixture was incubated for $15 \mathrm{~min}$ at room temperature and read at $540 \mathrm{~nm}$. $\mathrm{PGI}_{2}$ released by cultured ECs was detected by using a human $\mathrm{PGI}_{2}$ ELISA Kit (Sigma) according to the manual. Both $\mathrm{NO}$ and $\mathrm{PGI}_{2}$ were finally normalized to cell number.

\subsection{Smooth muscle cells (SMCs) culture}

SMC derived from human umbilical artery were isolated and cultured using the following method: the human umbilical cord was washed thoroughly with PBS to remove the blood outside, and then the artery was excised from the umbilical cord and opened at its length. Connective tissue outside and fibroblast layer outside were peeled off. The EC inside were gently scraped by a sharp tweezer. The muscle tissue was washed thoroughly with PBS and cut into small fragments. The fragments were then seeded in a single-used culture flask filled with $4 \mathrm{~mL}$ medium F12 and $1 \mathrm{~mL}$ fetal calf serum (FCS, Gibco BRL) mixture, and incubated at $37{ }^{\circ} \mathrm{C}$ in a humidified atmosphere containing $95 \%$ air and $5 \% \mathrm{CO}_{2}$. The fragments were removed after SMC migrated to the culture flask. Replicated cultures were performed by trypsinization when cells were approaching confluence. Cells were fed with freshly prepared growth medium every $24 \mathrm{~h}$. Subculture was performed when a degree of confluency $>80 \%$ was obtained, and cells were used between $2^{\text {nd }}$ and $5^{\text {th }}$ passage.

The sterilizing, seeding and staining procedure were the same as that of ECs, the concentration of SMC used for seeding was $5 \times 10^{4}$ cells per $\mathrm{mL}$.

\subsection{Statistical analysis}

Statistical significance was performed using one-way analysis of variance (ANOVA) using SPSS 17.0 (USA) statistical software. Data are presented as mean $\pm \mathrm{SD}$.

\section{Results}

\subsection{FTIR and ${ }^{1} \mathrm{H}$ NMR of PMMPC-HD}

As is shown in Fig. 2, PMMPC and cross-linked polymer PMMPC-HD were characterized by FTIR and ${ }^{1} \mathrm{H}$ NMR. The broad infrared absorption peak around $3450 \mathrm{~cm}^{-1}$ attributed to aromatic $-\mathrm{NH}-,-\mathrm{NH}_{2}$ and $\mathrm{O}-\mathrm{H}$ stretching vibrations. PMMPC and PMMPC-HD have lots of the groups in the branched chain. $2943 \mathrm{~cm}^{-1}$ attributed to $-\mathrm{CH}_{2}-$ stretching vibration which was in main and branch chains of the polymers. Both the two polymers had the group of $-\mathrm{C}=\mathrm{O}$ at the $1723 \mathrm{~cm}^{-1} .1656 \mathrm{~cm}^{-1}$ was ascribed to $-\mathrm{CONH}-$ in the polymer of PMMPC-HD. Furthermore, the appearance of $-\mathrm{CONH}-$ at $1546 \mathrm{~cm}^{-1}$ demonstrated that hexamethylene diamine was reacted with PMMPC. Simultaneously, the amino group was reacted with carboxyl of the polymer successfully. 1240, 1160 and $960 \mathrm{~cm}^{-1}$ were the absorbance of groups of MPC, corresponding to $-\mathrm{P}=\mathrm{O}$, $-\mathrm{P}-\mathrm{O}$ and $-\mathrm{N}^{+}\left(\mathrm{CH}_{3}\right)_{3}$. The existence of characteristic functional groups confirmed that the polymer of PMMPC-HD retain the properties of polyMPC. As is shown in ${ }^{1} \mathrm{H}$ NMR spectrum, at the chemical shift 4.7 ppm was $\mathrm{D}_{2} \mathrm{O}$ peak. The characteristic peak $-\mathrm{N}^{+}\left(\mathrm{CH}_{3}\right)_{3}$ was at $3.3 \mathrm{ppm}$, the 3.6, 4.0 and $4.3 \mathrm{ppm}$ was ascribed to the protons of the group of MPC, as shown in the Fig. 2 (h-i). Chemical shift of protons of methylene and methyl group which were marked with e and f was about 1.3 and $1.8 \mathrm{ppm}$. The appearances of chemical shift $\mathrm{a}, \mathrm{b}$ and $\mathrm{c}$ at about 2.8, 1.8 and $1.0 \mathrm{ppm}$, are also in consistent with the reported results ${ }^{35,36}$ and demonstrated that HD was grafted to the polymer PMMPC because of the existence of the proton of methylene in the PMMPC-HD which was ascribed to the HD.

\subsection{Characterization of Ti modified with PMMPC-HD and GREDVY}

Dopamine is a material easily self-polymerized and deposits onto numerous surfaces in wet conditions, the polished titanium (Ti) was immersed in dopamine solution for $48 \mathrm{~h}$. After micropatterned immobilization of PMMPC-HD and GREDVY, SEM, water contact angle and FTIR characterization were proceed. As shown in Fig. 3(a and b), SEM and optional graph of the micropattern of Ti-PDA-M/R(P) was complete, neat and orderly. The width of bands was $25 \mu \mathrm{m}$ on average.

The stability of the micropattern mainly depends on the PMMPC-HD, the sample of Ti-PDA-M(P) was immersed in 


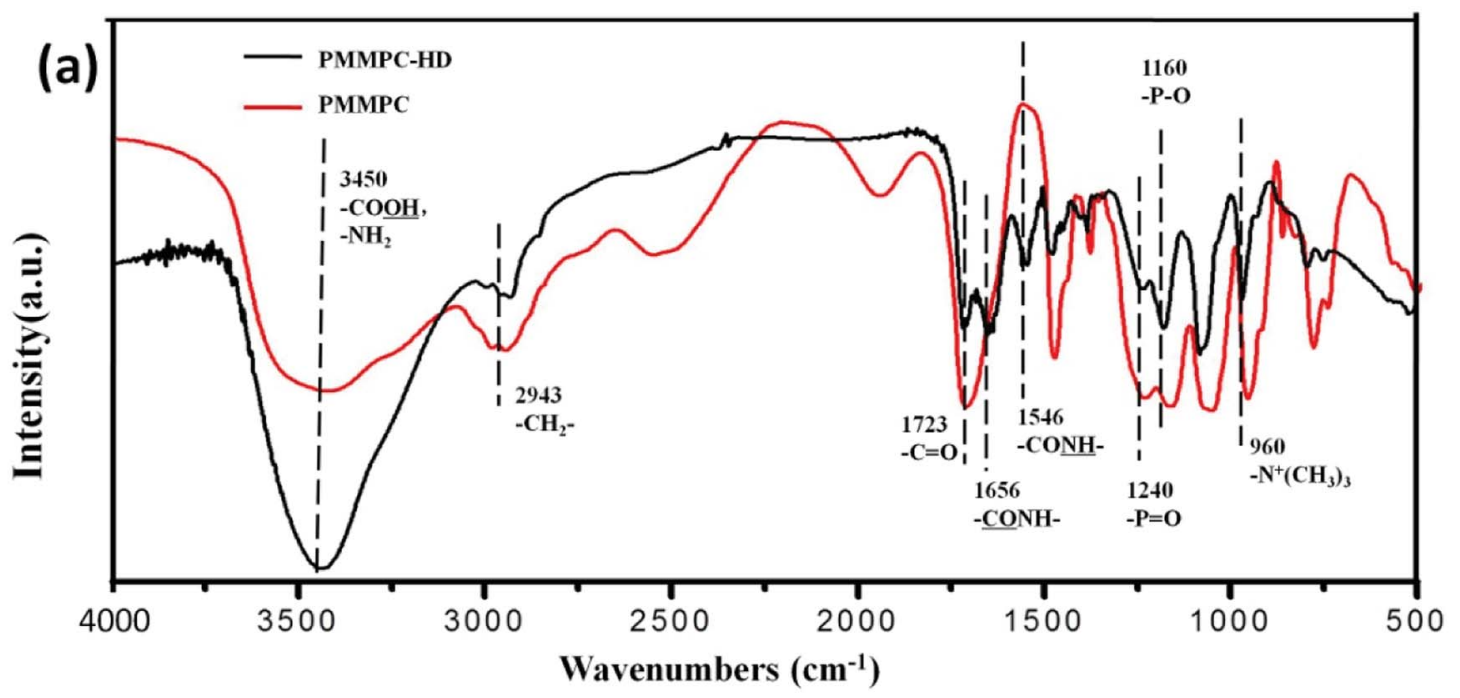

(b)
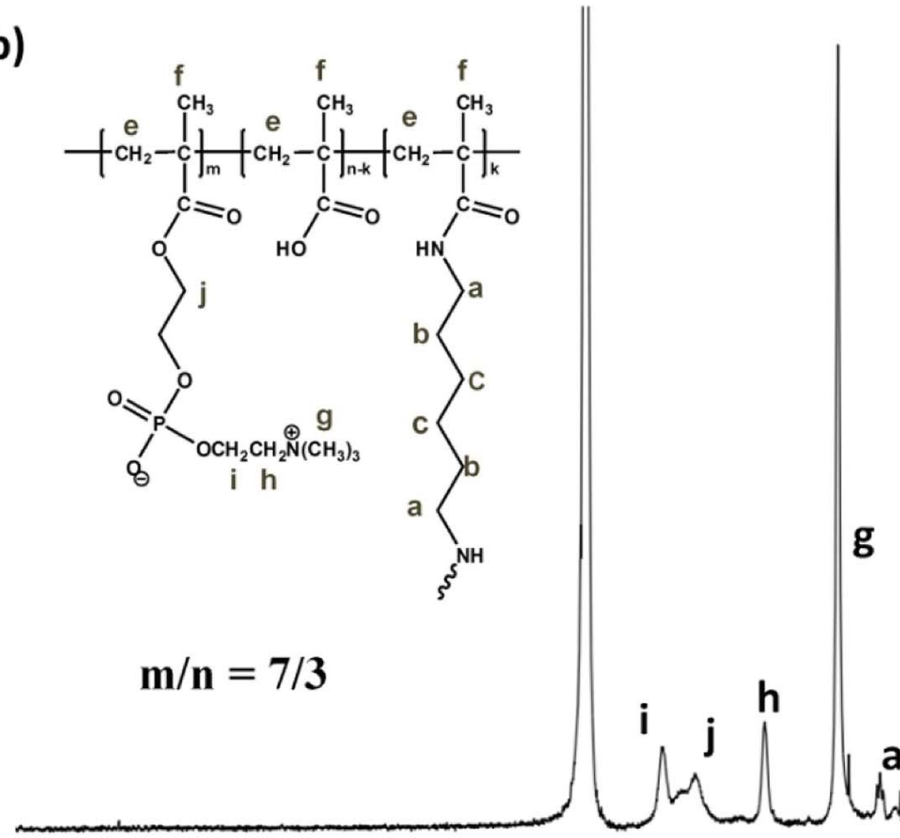

$\mathbf{m} / \mathbf{n}=7 / 3$

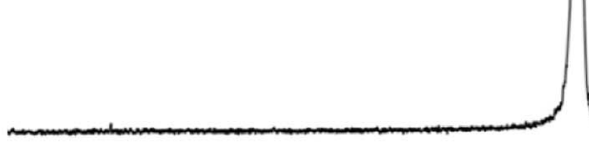

Chemical shift (ppm)

Fig. 2 FTIR (a) spectra of PMMPC and PMMPC-HD and ${ }^{1} H$ NMR (b) of structural unit of PMMPC-HD.

deionized water for $1,3,5$ and 7 days. The micropattern of PMMPC-HD was still visible on the surface of polydopamine coating (Fig. S1 $\dagger$ ). The data demonstrated that the micropattern was stable enough to regulate cells. The thickness of PMMPCHD pattern detected after immersed in water and PBS (pH: 7.4) for 1, 3, 5 and 7 days. As shown in Fig. S2, $\uparrow$ the thickness of Ti-PDA-M(P) micropattern increased because of swelling effect in deionized water and PBS. The thickness of 1 day and 3 days in deionized water was $220 \mathrm{~nm}$, but the thickness of 5 days increase to $730 \mathrm{~nm}$. The data showed that swelling effect of 1 and 3 days was not obviously. The thickness of Ti-PDA-M(P) micropattern in PBS (pH: 7.4) increased slowly from $308 \mathrm{~nm}$ to $410 \mathrm{~nm}$. The data suggested influence of ion strength on the Ti-PDA-M(P) micropattern was obviously, because ion concentration and ion strength of PBS solution were bigger than those of deionized water. It can be concluded that PBS buffer ( $\mathrm{pH}$ 7.4) containing a great many ions can accelerate hydrolysis of PMMPC-HD.

As is shown in Fig. 4, the results of water contact angle showed that the sample Ti-PDA-M(P), Ti-PDA-M/R(P) was more 

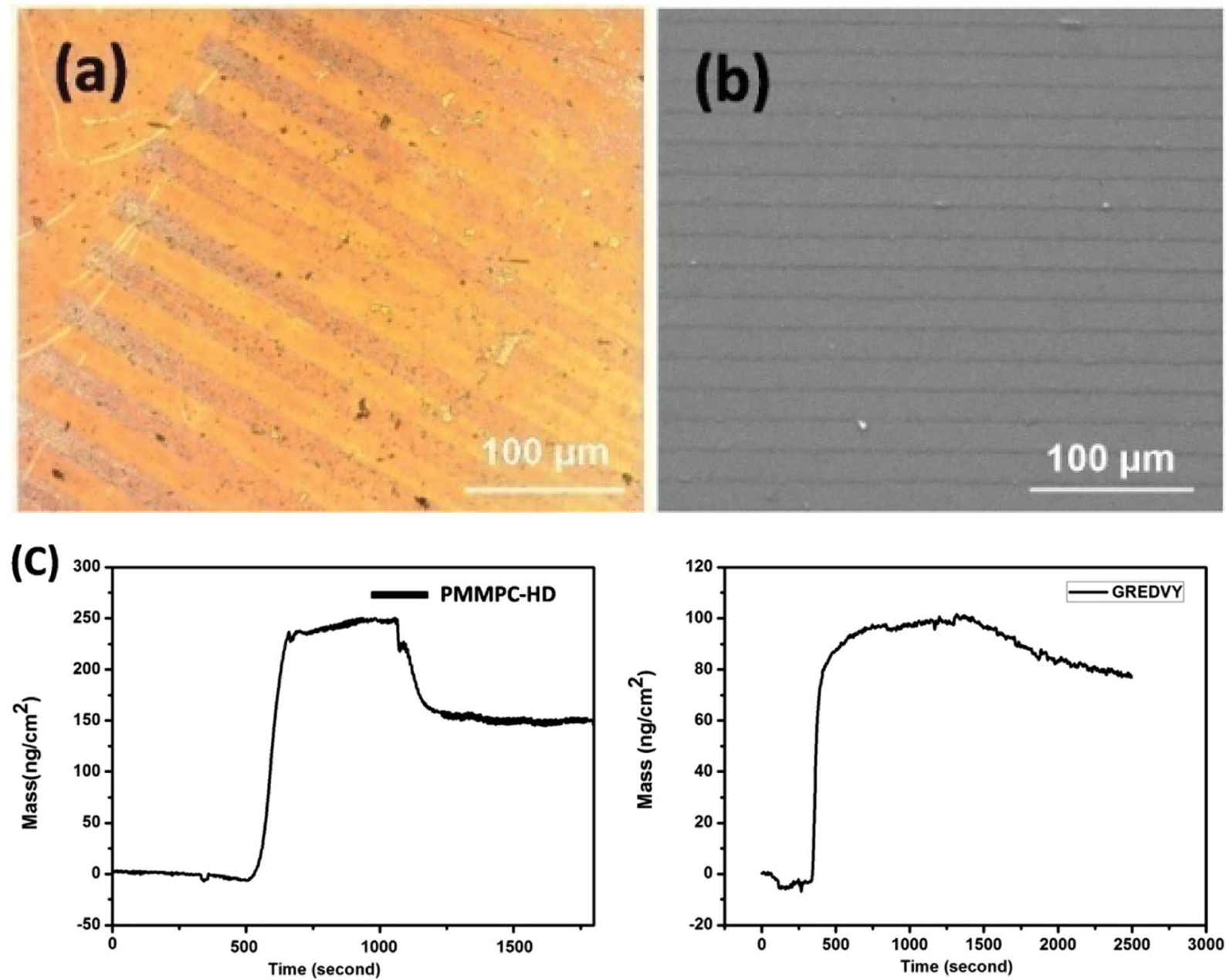

Fig. 3 Optional graph (a) and SEM image (b) of the sample Ti-PDA-M/R(P). Adsorption and detachment process of PMMPC-HD and GREDVY on PDA-modified Au QCM sensor (Au-PDA) (c).

hydrophilic than the Ti and Ti-PDA, whose degree decreased to $46.2 \pm 1.53^{\circ}$, because PMMPC-HD on the surface containing phosphorylcholine (PC) groups has super hydrophilic property.

The QCM-D results showed that the amount of PMMPC-HD could be immobilized onto PDA coating, and it reached $137.7 \pm$ $2.8 \mathrm{ng} \mathrm{cm}^{-2}$. From the data, it can be concluded that the polymer can adhere onto PDA coating covalently by means of condensation reaction. Similarly, the peptide GREDVY, which has the free amino, carboxyl and phenyl groups can be immobilized on PDA coating through chemical reaction, electrostatic interaction and $\pi-\pi$ Interactions, and the amount of GREDVY on the surface reached $80.0 \pm 2.4 \mathrm{ng} \mathrm{cm}^{-2}$.

The surface infrared grazing FTIR of samples showed that the groups in surface coatings. As shown in Fig. 5 , all coatings had a broad peak around $3400 \mathrm{~cm}^{-1}$ ascribed to $-\mathrm{OH}$ and $-\mathrm{NH}_{2}$ stretching vibrations, which exits in the polydopamine coating, PMMPC-HD and GREDVY. Three coatings also presented peaks at $2950 \mathrm{~cm}^{-1}$ (aliphatic C-H stretching vibrations of $\mathrm{CH}_{2}$ ). The peak at $1740 \mathrm{~cm}^{-1}$ was ascribed to the $\mathrm{C}=\mathrm{O}$ stretching vibrations of $-\mathrm{COOH}$ and $-\mathrm{COOR}$ groups, which were in the coating Ti-PDA-M/R(P) and Ti-PDA-M(P), but not the PDA coating. The

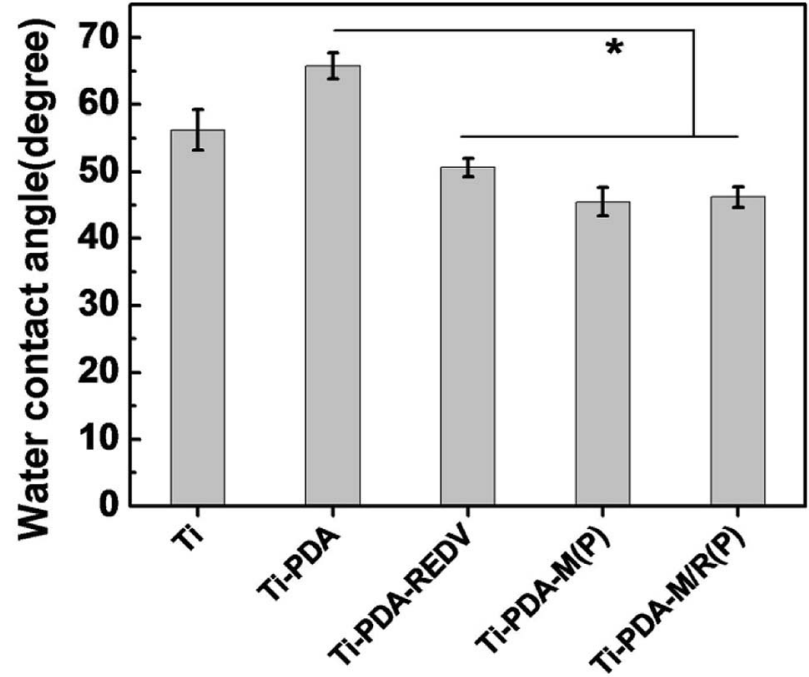

Fig. 4 Water contact angle of samples. Data presented as mean $\pm S D$, $n=6 .{ }^{*} p<0.05$. 




Fig. 5 FTIR spectra of samples: Ti-PDA, Ti-PDA-M(P) and Ti-PDA-M/R(P).

difference showed that polymer of PMMPC-HD was successfully immobilized on the PDA coating. As is shown in figure of partial enlargement, PDA coating had the peak at 1635 and $1629 \mathrm{~cm}^{-1}$ ascribed to $\mathrm{C}=\mathrm{C}-\mathrm{C}=\mathrm{O}$ (aliphatic $\mathrm{C}=\mathrm{C}$ stretching vibrations) and the peak at $1516 \mathrm{~cm}^{-1}$ attributed to $\mathrm{C}-\mathrm{N}$ stretching vibrations. The peak around $1661 \mathrm{~cm}^{-1}$ was ascribed to amide I band $(-\mathrm{CONH}-, \mathrm{I})$, and amide II band $(-\mathrm{CONH}-$, II $)$ was at $1546 \mathrm{~cm}^{-1}$, both of which were found in Ti-PDA-M/R(P) and TiPDA-M(P). Additionally, the Ti-PDA-M/R(P) had the peak of 1658 and $1640 \mathrm{~cm}^{-1}$, so it can be concluded that the peaks were stretching vibration of $\mathrm{C}=\mathrm{C}$ of phenyl (with hydroxyl group) in GREDVY, proving that the peptide was successfully immobilized on PDA coating.

\subsection{Platelets adhesion and whole blood adhesion}

The in vitro platelet adhesion test is used to investigate the blood compatibility of biomaterials. To study adhesion of platelets on different samples, at least nine sight fields with the size of $100 \times 100 \mu \mathrm{m}$ were collected through a fluorescence microscope and were counted adherent platelets for statistical analysis. The quantity of platelets on all samples after contact with PRP for 15 min is presented in Fig. 6. The data showed that Ti-PDA facilitated a much higher level of platelet adhesion compared with samples of Ti, Ti-PDA-REDV, Ti-PDA-M(P) and Ti-PDA-M/R(P). The quantity of adhered platelets on all the samples decreased in the order: Ti-PDA $>\mathrm{Ti}>\mathrm{Ti}$-PDA-REDV $>$ TiPDA-M $(\mathrm{P})>$ Ti-PDA-M/R(P). On the Ti-PDA surface the platelets were in aggregation to a greater extent. In the same way, the band containing PDA surface in samples of Ti-PDA-M(P) also aggregated the adhered platelets. On the Ti-PDA-REDV surface, the number of adhered platelets decreased and the degree of aggregation weakened. On the Ti-PDA-M/R(P) surface, the platelets were least of all and the degree of aggregation became weaker.

As is shown in Fig. 7, on the surface of Ti and Ti-PDA, the number of adhered red blood cells is the largest of all. Also, the adhered platelets on the Ti-PDA were more than other samples. But on the Ti-PDA-REDV, the amount of adhered red blood cells decreased obviously, while the platelets on Ti-PDA-REDV also reduced significantly. But on the surface of Ti-PDA-M(P), the platelets were adhered on the edge of micropattern and activated, simultaneously the platelets were mainly dendritic. Only few red blood cells were adhered. On the surface of Ti-PDA-M/ $R(P)$, very few platelets and red blood cells were adhered, and the platelets were round and the red blood cells were like round flat cakes, displaying normal phenotype in vivo. The data of whole blood adhesion showed the polyMPC had good hemocompatibility.

\subsection{EC culture}

In vitro, the ECs in culture medium are in disorder and the microenvironment of cells is isotropic. On the micropatterned surface, the cells can be regulated. As shown in Fig. 8(a), the cells on surface of Ti, Ti-PDA and Ti-PDA-REDV without micropattern were distributed freely, and the cell morphology was ellipsoidal. On the Ti-PDA-M(P) and Ti-PDA-M/R(P), the cells were highly ordered and elongated. The long axis of the cells was in the direction of stripe of the micropattern. It is noteworthy that axial ratio of the cells on the surface with micropattern was maximum to 8 and the cells on Ti-PDA-M/R(P) begin interconnecting by the third day. Also it can be seen that the cells on the surface with peptide REDV were better in morphology than those on Ti and Ti-PDA. Considering PMMPCHD was resistance to proteins and cells, the data suggested that REDV can capture ECs. Quantification of the degree of cell elongation (elongation factor), which we defined as the length of the longest axis divided by the length of the short axis across the cell nucleus, showed that ECs on micropattern exhibited a significantly higher degree of elongation compared with ECs on flat samples.

Fig. 8(b) showed the amount of ECs on the surface of samples. The average number of ECs on surfaces of samples was counted at least nine sight fields. It can be seen that after being cultured for 1 day, the number of cells on Ti-PDA-M(P) was smallest of all, but on the Ti-PDA-M/R(P) the number increased obviously. On the 3-third day, the amount of ECs of all 

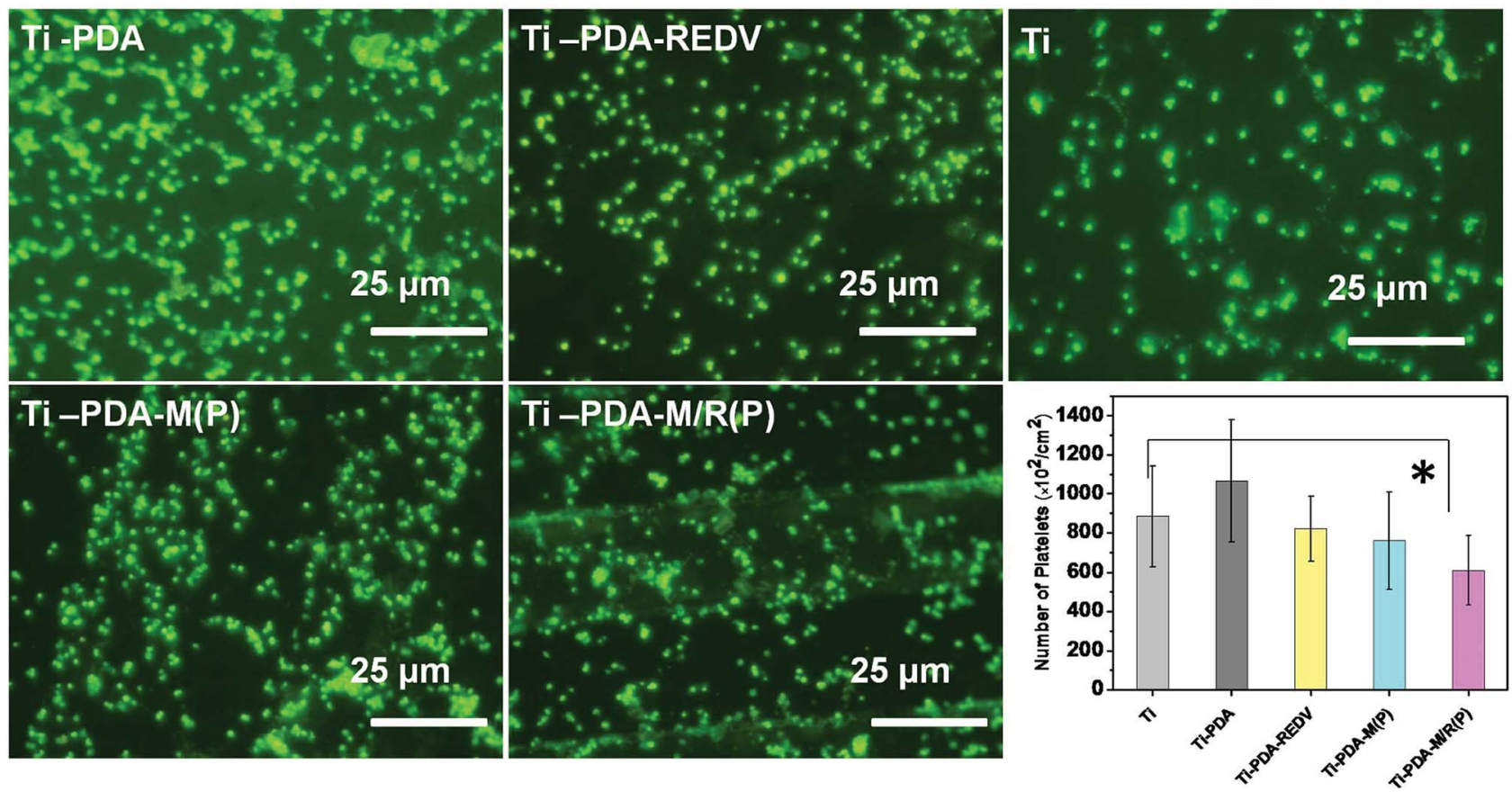

Fig. 6 Fluorescence images and number of adherent platelets on the surface of samples. Data presented as mean $\pm \mathrm{SD}, n=9 .{ }^{*} p<0.05$.

samples and surface coverage increased, and on Ti-PDA-M/R(P) the number was bigger than that of Ti-PDA-M(P). On the Ti-PDA surface, the EC spreading was on a smaller scale than that on the Ti-PDA-REDV. That is to say, the peptide REDV can enhance adhesion and proliferation of ECs and is beneficial to EC spreading compared to Ti and PDA coating.

\subsection{Release of NO and PGI ${ }_{2}$ of ECs}

Healthy ECs are essential to maintain vascular homeostasis by the continuous release of $\mathrm{NO}$ and $\mathrm{PGI}_{2}$. $\mathrm{NO}$ and $\mathrm{PGI}_{2}$ are predominant products that act as antiproliferative agents in vascular smooth muscle cells. ${ }^{37}$ As is shown in Fig. 9, the determinations of $\mathrm{NO}$ and $\mathrm{PGI}_{2}$ release levels per cell were performed to evaluate EC functions. ECs cultured with Ti-PDA-
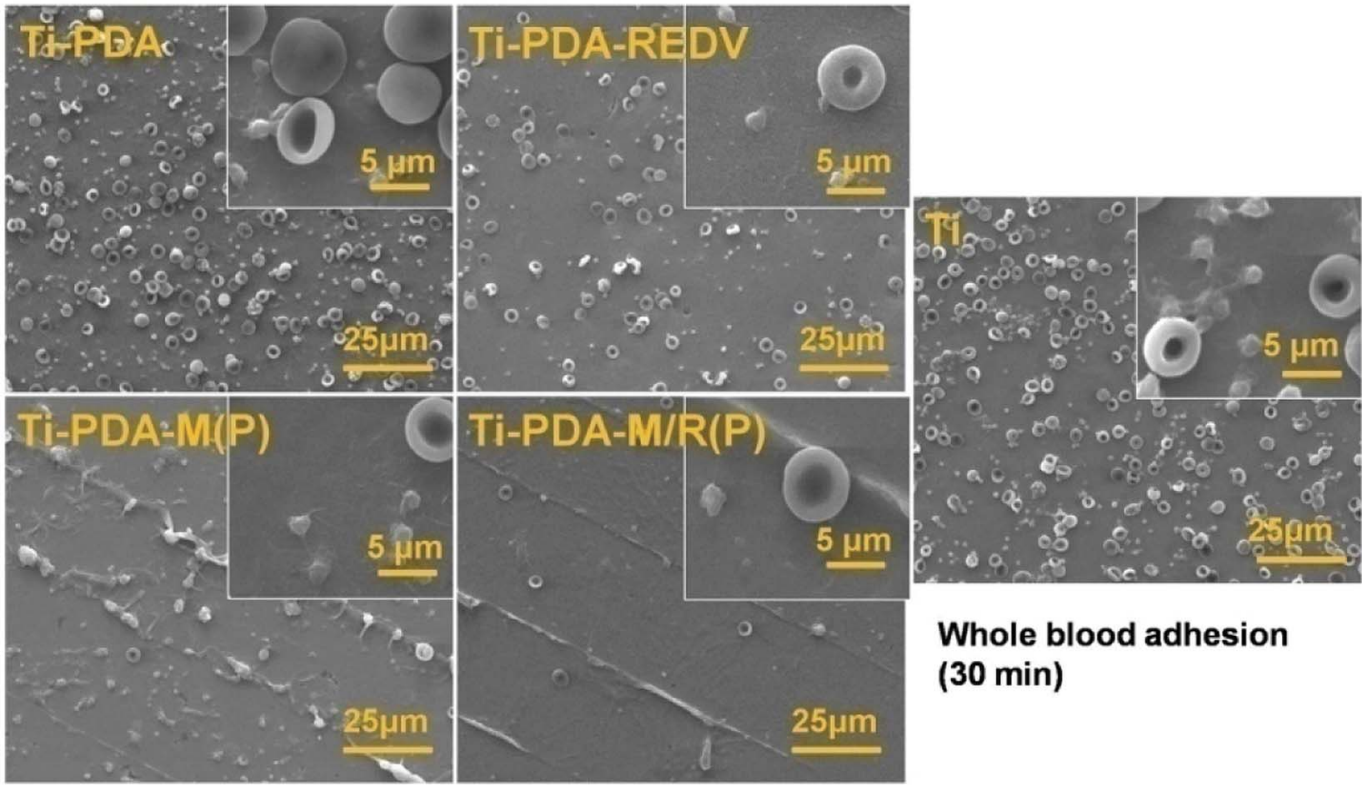

Whole blood adhesion (30 min)

Fig. 7 SEM images of whole blood adhesion (30 min) on surfaces of samples. 

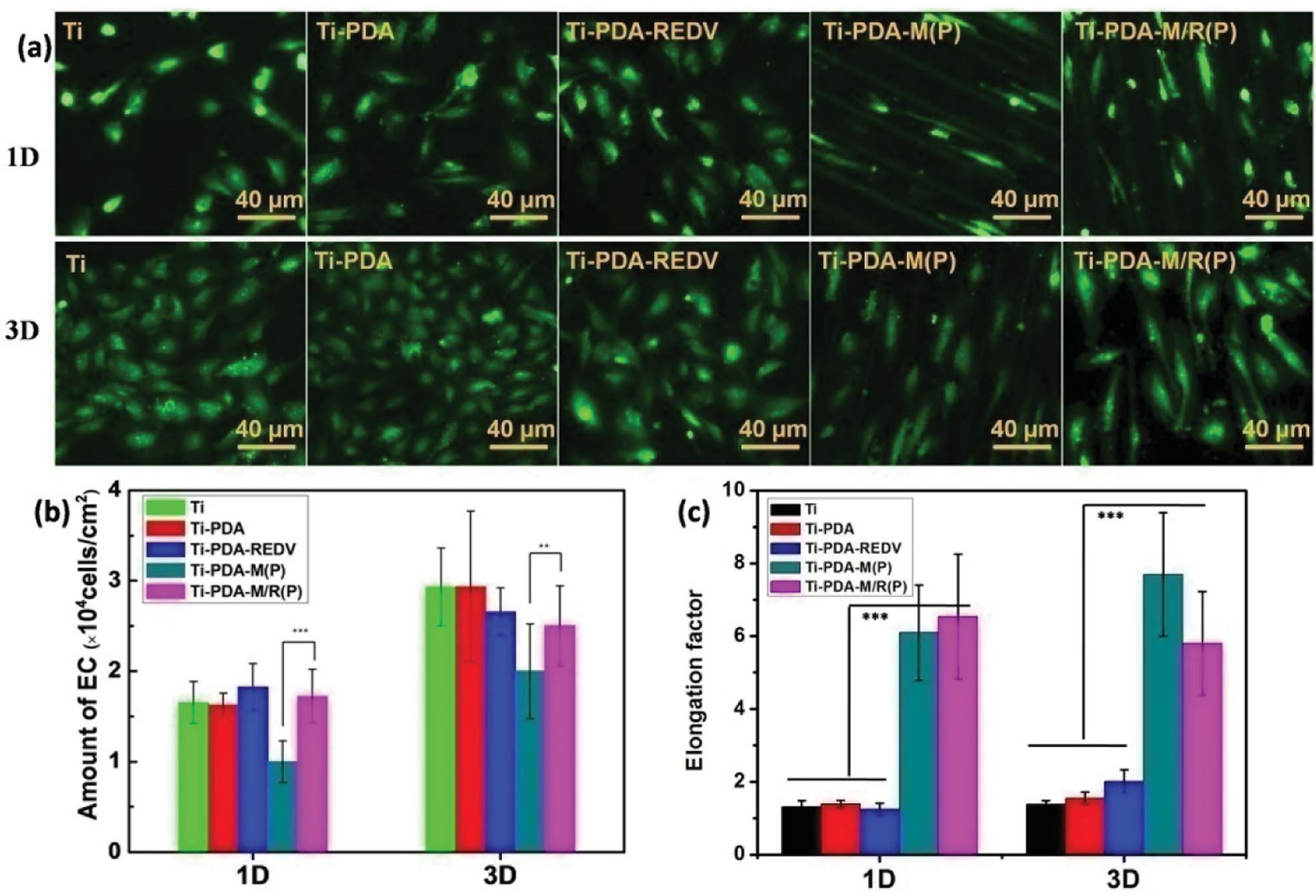

Fig. 8 Fluorescent images of ECs on the samples after 1 day and 3 days of culture (a); amount of ECs was counted at least nine sight fields (b), elongation factor of EC on surface of samples (c). Data presented as mean $\pm \mathrm{SD}, n=9 . * * p<0.01, * * * p<0.001$.

$\mathrm{M}(\mathrm{P})$ and Ti-PDA-M/R(P) produced more $\mathrm{NO}$ and $\mathrm{PGI}_{2}$ than that of Ti and Ti-PDA by the $1^{\text {st }}$ and $3^{\text {rd }}$ day, demonstrating that micropattern can regulate EC functions. Considering that the amount of ECs on Ti and Ti-PDA surface were bigger than that on micropattern samples, the elongated cells showed better cell functions. With Ti-PDA-REDV, EC had larger spreading area compared to that with Ti and Ti-PDA, and displayed better cell functions.

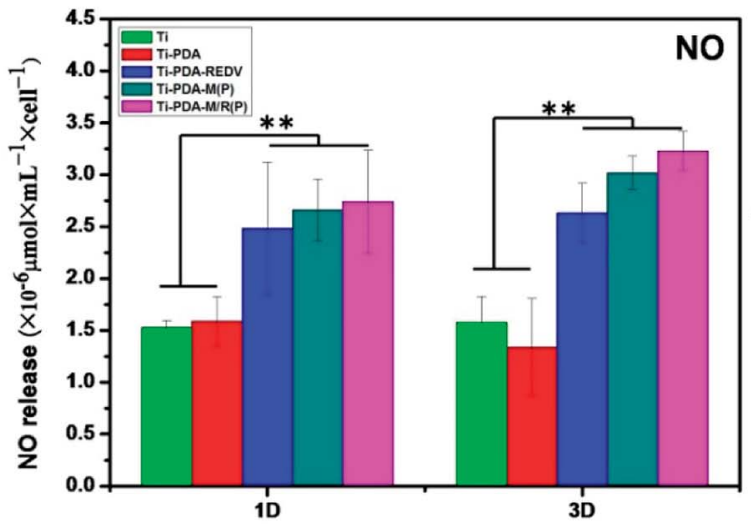

\subsection{SMCs culture}

For the aim of improving the effect of antiproliferation of vascular smooth muscle cells, micropattern had the property of elongating the cells ${ }^{33}$ and enhancing expressing of smooth muscle $\alpha$-actin of SMCs which demonstrates a phenotype shift to a more contractile phenotype. ${ }^{38}$ As shown in Fig. 10, on the surface of Ti and Ti-PDA after 1 day and 3 days of culture, most cells were in random distribution, and the cell morphology was spread. The average number of SMCs on surfaces of samples



Fig. $9 \mathrm{NO}$ and $\mathrm{PGl}_{2}$ release in culture media for samples (the size of sample: $\Phi 10 \mathrm{~mm}, n=3$ ). Data presented as mean $\pm \mathrm{SD}, * p<0.05, * * p<0.01$. 
was counted at least nine sight fields. As shown in Fig. 10(b), the amount of smooth muscle cells on Ti and Ti-PDA after 3 days of culture was larger than that of 1 day. On the surface of Ti-PDAREDV, the number of cells after 1 day of culture was fewer than that of Ti-PDA, but had not obvious difference after 3 days of culture. It can be speculated that the PDA coating was not completely covered by GREDVY, in other words, PDA coating was exposed to adhere SMCs on the surface of Ti-PDA-REDV. On the surface of micropattern, Ti-PDA-M(P) and Ti-PDA-M/R(P), the cells were distributed orderly, with the long axis of cells in the direction of micropattern. The amount of cells on Ti-PDA$\mathrm{M}(\mathrm{P})$ of a 3 days culture was larger than that of 1 day, similarly, SMCs of a 3 days culture on the surface of Ti-PDA-M/R(P) was more than that of the 1 day culture. Moreover, the number of SMCs adhered on Ti-PDA-M/R(P) was smaller than that of other sample after 3 days culture. The results suggest that the micropattern of Ti-PDA-M/R(P) inhibited proliferation of smooth muscle cell to a certain extent. The conclusion was consistent with the literature. ${ }^{33,38}$ As shown in Fig. 10(c), the ratio of ECs/SMCs' numbers on samples was 4, 5 after 1 day culture. The data suggested that ECs were easier to adhere on samples than SMCs. After 3 days culture, the ratio of Ti, Ti-PDA and Ti-PDA-REDV was about 2-3, but that of Ti-PDA-M(P) and Ti-PDA-M/R(P) increased significantly. The ratio of Ti-PDA-M/ $\mathrm{R}(\mathrm{P})$ was also bigger than that of Ti-PDA-M (P). The data suggested proliferation of SMCs was quicker than that of ECs, but the ratio of Ti-PDA-M/R(P) could demonstrate the competitive growth of ECs over SMCs could be increased and suggested the GREDVY induced the adhesion of the EC cells.

\section{Discussion}

Micropatterning is an important method for regulating the adherent cells including vascular smooth muscle cells, endothelial cells and so on. The micro-transfer molding method was chosen to fabricate micropattern with the PDMS stamp to adhere PMMPC-HD onto the surface, the optical and SEM image showed a strip interval distribution. The IR and ${ }^{1} \mathrm{H}$ NMR results demonstrated that amino group reacted with carboxyl group. The QCM showed that PMMPC-HD can be immobilized onto PDA coatings mainly through condensation reaction of amino and carboxyl. Likewise, the peptide GREDVY containing amino and carboxyl groups can be immobilized. The Ti-PDA-M/ $\mathrm{R}(\mathrm{P})$ micropattern of PMMPC and GREDVY was successfully
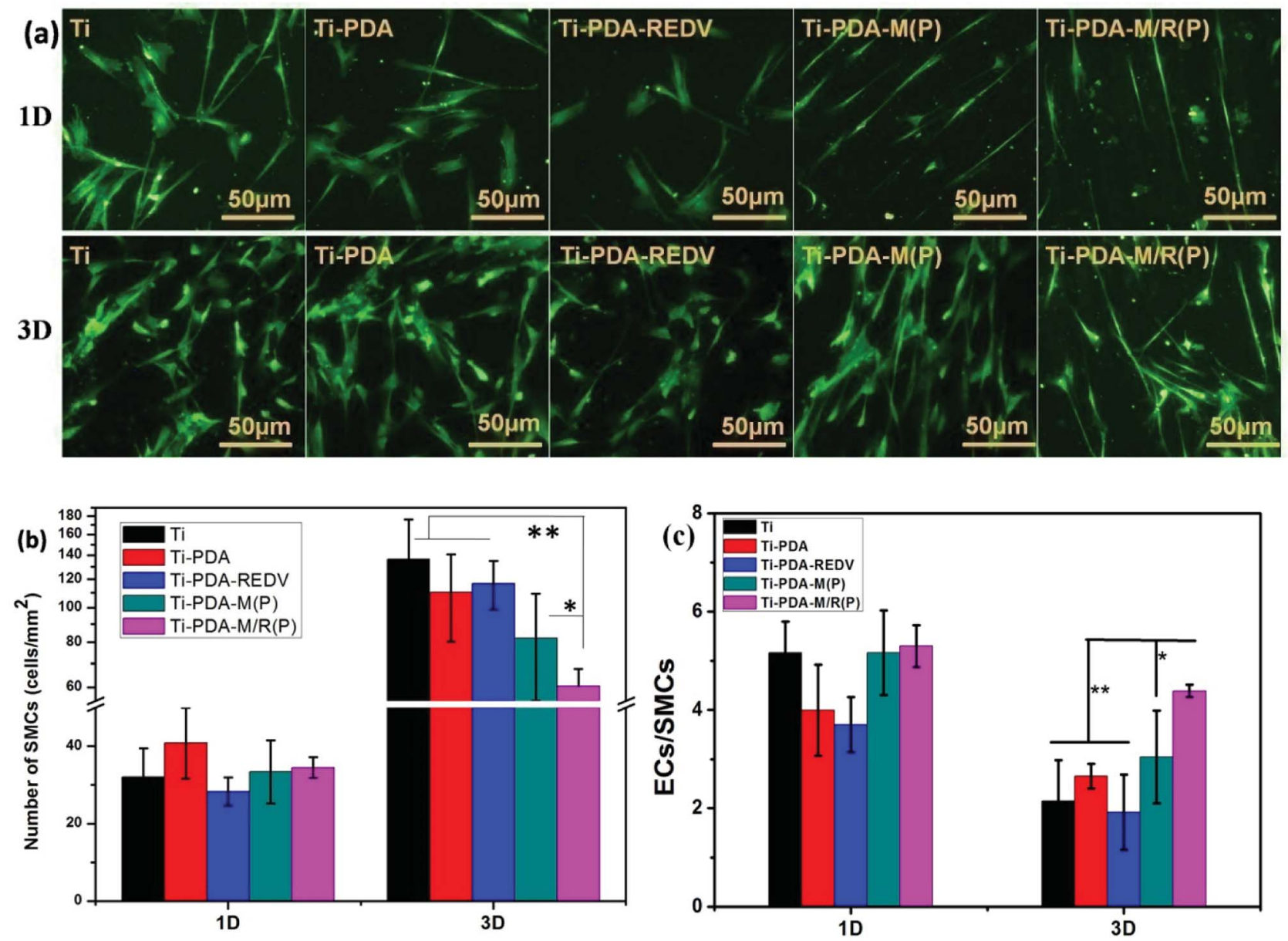

Fig. 10 Fluorescent images of SMCs on the samples after 1 day and 3 days of culture (a), number of SMCs (b) was counted at least nine sight fields, (c) ratios of ECs/SMCs' numbers on samples. Data presented as mean $\pm \mathrm{SD}, n=9, * p<0.05, * * p<0.01, * * * p<0.001$. 
fabricated and the ECs' functions were successfully regulated. $\mathrm{NO}$ and $\mathrm{PGI}_{2}$ release of the elongated ECs were improved.

The micropattern of polyMPC has been fabricated using micropatterned mask. Generally, the preparation method is as follows: first, the MPC-polymer was coated on the surface uniformly, and then UV or $\mathrm{O}_{2}$ plasma was used to initiate a photochemical reaction or etch the polymer ${ }^{7}$ through micropatterned mask. Last, remove the polymer which can't be immobilized firmly and the regular micropattern was fabricated. The reaction which UV initiate in the above steps contains azidonation reaction ${ }^{39}$ and decomposing molecular layer. ${ }^{40}$ The area of PMMPC is nonspecific suppression adsorption of proteins, simultaneously the area of substrate can adhere amounts of proteins in vitro and adhere platelets, cells. The REDV peptide was chosen because this short peptide sequence is known to be selectively adhered for ECs. ${ }^{28,41}$ To immobilize special peptide GREDVY onto the Ti, we chose PDA coating containing quinone groups, amino and carboxyl groups as a bridge to connect amino and carboxyl group of GREDVY. To fabricate the micropattern, PDMS was used to construct micropattern is convenient and doesn't require critical reaction conditions. The reaction can occur at room temperature in humid environment. Considering polyMPC which have excellent cytocompatibility without any cytotoxicity ${ }^{42}$ it is likely to apply in three-dimensional print field for fabricating micropattern and tissue engineering. In all, polyMPC with micropattern have good hemocompatibility ${ }^{43}$ can be potential in vascular tissue engineering.

As was mentioned above, in order to capture EC selectively, the peptide GREDVY was chosen to immobilize onto PDA coating. The peptide arginine-glycine-aspartate (RGD), which is the principal integrin-binding present within ECM proteins such as fibronectin, vitronectin, fibrinogen, osteopontin, and bone sialoprotein, ${ }^{\mathbf{4 4 , 4 5}}$ is recognized by platelet integrin receptors which promote their adhesion, ${ }^{46,47}$ and enhance smooth muscle cell adhesion. ${ }^{48}$ So the RGD can adhere to smooth muscle cells and platelets and the peptide REDV cannot allow specific adhesion as ligand-integrin interactions. But the result of the platelets adhesion showed that amount of platelets on TiPDA-REDV decreased slightly and degree of aggregation weakened. It can be concluded that PDA coating ${ }^{13}$ had a certain degree of exposure which can lead to activation and aggregation of adhered platelets. It might be caused by the large amount of primary amino groups (positively charged) that are likely to promote platelet adhesion (negatively charged) by electrostatic interaction. $^{49,50}$ Some anticoagulant biomolecules ${ }^{51-53}$ and polymer (polyethylene glycol ${ }^{54}$ and polyMPC $^{13}$ ) are used to improve hemocompatibility. All in all, the sample of Ti-PDAREDV can adhere to endothelial cells selectively, whose morphology of EC was better than that of ECs on Ti-PDA. The hemocompatibility of Ti-PDA-REDV had improved to some extent, but it needs further improvement.

NO release can be promoted by shear stress with defined direction. ${ }^{55-57}$ It can be concluded that micropattern which has the similar functions might influence the integrin and morphology, which induce the synthesis of endothelial NO synthase (eNOS) which was contributing to NO release of ECs.
Similarly, the $\mathrm{PGI}_{2}$ release be also upregulated by shear stress, ${ }^{58}$ and micropattern can promote the $\mathrm{PGI}_{2}$ secretion of ECs. ${ }^{59}$ The NO release plays a crucial role in governing endothelial function and health, inhibition of platelet adhesion and activation and inhibition of smooth muscle proliferation and migration. ${ }^{60}$ Promoting $\mathrm{NO}$ and $\mathrm{PGI}_{2}$ by micropattern is a good way of regulating some EC functions as shear stress in vivo, which can improve the vasodilation and prevent the formation of atherosclerosis.

From previous studies, the thickness of polymer MPC was about 10-40 nm, ${ }^{40,61,62}$ whose maximum reached about $100 \mathrm{~nm},{ }^{63}$ by surface initiated atom transfer radical polymerization. As is called the "grafting from" way, the degree of polymerization was about 200 . The "grafting to" way, as is reported, the thickness was about $800 \mathrm{~nm}$, the top thickness reached 4000 after polyMPC incubated in water for $60 \mathrm{~min} \cdot{ }^{39}$ The molecular weight of the polymer reached $2.2 \times 10^{5}$. It came into a conclusion that the thickness on the surface was positive correlation with molecular weight of MPC polymer. Considering the molecular weight of PMMPC (170 kDa), the thickness might be not enough to impact EC in the third dimension. Since the two-dimensional micropattern can regulate cell adhesion and distribution, morphology and functions in vitro to some extent, and make the cell present highly ordered, the cell microenvironment in vivo was even more complicated and therefore has more effective influences on cells. As a result, attempting to fabricate three-dimensional is a promising way to regulate cell functions.

\section{Conclusion}

Cross-linked PMMPC-HD with hexamethylenediamine was synthesized successfully and immobilized on PDA coating using PDMS stamps, and peptide GREDVY can be immobilized onto PDA coating because PMMPC-HD is non-specific resistance adhesion of proteins. The Ti-PDA-M/R(P) micropattern was successfully fabricated with PMMPC-HD and GREDVY. Platelets adhesion and whole blood adhesion showed that polyMPC has good hemocompatibility and on the peptide GREDVY coated surface the degree of platelet aggregation weakened and the number decreased. The micropattern Ti-PDA-M/R(P) can regulate EC morphology, orientation and functions. Upregulation of $\mathrm{NO}$ and $\mathrm{PGI}_{2}$ release demonstrated that elongated $\mathrm{EC}$ was closer to native endothelial cells.

\section{Conflicts of interest}

There are no conflicts to declare.

\section{Acknowledgements}

This work was supported by financially supported by the National Natural Science Foundation of China (No. 81330031, 31570963), National Key Research and Development Program of China (2016YFC1100402), Key Basic Research Project (No. 2011CB606204). 


\section{References}

$1 \mathrm{~F} . \mathrm{Xu}, \mathrm{Y}$. Sun, Y. Chen, et al., Endothelial Cell Apoptosis is Responsible for the Formation of Coronary Thrombotic Atherosclerotic Plaques, Tohoku J. Exp. Med., 2009, 218, 2533.

2 D. E. Anderson and M. T. Hinds, Endothelial cell micropatterning: methods, effects, and applications, Ann. Biomed. Eng., 2011, 39, 2329-2345.

3 M. A. Gimbrone and G. García-Cardeña, Endothelial cell dysfunction and the pathobiology of atherosclerosis, Circ. Res., 2016, 118, 620-636.

4 P. Zorlutuna, N. Annabi, G. Camci-Unal, et al., Microfabricated Biomaterials for Engineering 3D Tissues, Adv. Mater., 2012, 24, 1782-1804.

5 D. E. J. Anderson and M. T. Hinds, Endothelial Cell Micropatterning: Methods, Effects, and Applications, Ann Biomed Eng., 2011, 39, 2329-2345.

6 F. Zhang, G. Li, P. Yang, et al., Fabrication of biomoleculePEG micropattern on titanium surface and its effects on platelet adhesion, Colloids Surf., B, 2013, 102, 457-465.

7 K. Kuribayashi, Y. Tsuda, H. Nakamura, et al., Micropatterning of phosphorylcholine-based polymers in a microfluidic channel, Sens. Actuators, B, 2010, 149, 177183.

8 N. F. Huang, E. S. Lai, A. J. Ribeiro, et al., Spatial patterning of endothelium modulates cell morphology, adhesiveness and transcriptional signature, Biomaterials, 2013, 34, 29282937.

9 H. M. Khanlou, B. C. Ang, M. M. Barzani, et al., Prediction and characterization of surface roughness using sandblasting and acid etching process on new non-toxic titanium biomaterial: adaptive-network-based fuzzy inference system, Neural Comput. Appl., 2015, 26, 1751-1761.

10 M. Kulkarni, A. Mazare, P. Schmuki, et al., Biomaterial Surface Modification Of Titanium and Titanium Alloys for Medical Applications, Nanomedicine, 2014, 5, 111-136.

11 X. Liu, P. K. Chu and C. Ding, Surface modification of titanium, titanium alloys, and related materials for biomedical applications, Mater. Sci. Eng., R, 2004, 47, 49121.

12 Y. Yao, K. Fukazawa, W. Ma, et al., Platelet adhesionresistance of titanium substrate with mussel-inspired adhesive polymer bearing phosphorylcholine group, Appl. Surf. Sci., 2012, 258, 5418-5423.

13 W. Ma, P. Yang, J. Li, et al., Immobilization of poly (MPC) brushes onto titanium surface by combining dopamine self-polymerization and ATRP: Preparation, characterization and evaluation of hemocompatibility in vitro, Appl. Surf. Sci., 2015, 349, 445-451.

$14 \mathrm{M}$. Kyomoto and K. Ishihara, Self-initiated surface graft polymerization of 2-methacryloyloxyethyl phosphorylcholine on poly(ether ether ketone) by photoirradiation, ACS Appl. Mater. Interfaces, 2009, 1, 537542 .
15 W. Feng, X. Gao, G. McClung, et al., Methacrylate polymer layers bearing poly(ethylene oxide) and phosphorylcholine side chains as non-fouling surfaces: In vitro interactions with plasma proteins and platelets, Acta Biomater., 2011, 7, 3692-3699.

$16 \mathrm{~J}$. Wu, W. Lin, Z. Wang, et al., Investigation of the Hydration of Nonfouling Material Poly(sulfobetaine methacrylate) by Low-Field Nuclear Magnetic Resonance, Langmuir, 2012, 28, 7436-7441.

17 I. Banerjee, R. C. Pangule and R. S. Kane, Antifouling Coatings: Recent Developments in the Design of Surfaces That Prevent Fouling by Proteins, Bacteria, and Marine Organisms, Adv. Mater., 2011, 23, 690-718.

18 W. Feng, S. P. Zhu, K. Ishihara, et al., Adsorption of fibrinogen and lysozyme on silicon grafted with poly(2methacryloyloxyethyl phosphorylcholine) via surfaceinitiated atom transfer radical polymerization, Langmuir, 2005, 21, 5980-5987.

19 W. Feng, J. Brash and S. P. Zhu, Atom-transfer radical grafting polymerization of 2-methacryloyloxyethyl phosphorylcholine from silicon wafer surfaces, J. Polym. Sci., Part A: Polym. Chem., 2004, 42, 2931-2942.

20 Y. C. Zhao, Q. F. Tu, J. Wang, et al., Crystalline $\mathrm{TiO}_{2}$ grafted with poly(2-methacryloyloxyethyl phosphorylcholine) via surface-initiated atom-transfer radical polymerization, Appl. Surf. Sci., 2010, 257, 1596-1601.

21 Y. Iwasaki, A. Matsunaga and S. Fujii, Preparation of Biointeractive Glycoprotein-Conjugated Hydrogels through Metabolic Oligosacchalide Engineering, Bioconjugate Chem., 2014, 25, 1626-1631.

22 X. Liu, H. Li, Y. Chen, et al., Mixed-Charge Nanoparticles for Long Circulation, Low Reticuloendothelial System Clearance, and High Tumor Accumulation, Adv. Healthcare Mater., 2014, 3, 1439-1447.

23 Y. Wei, Y. Ji, L. L. Xiao, et al., Surface engineering of cardiovascular stent with endothelial cell selectivity for in vivo re-endothelialisation, Biomaterials, 2013, 34, 2588-2599.

24 S. P. Massia and J. A. Hubbell, Vascular endothelial cell adhesion and spreading promoted by the peptide REDV of the IIICS region of plasma fibronectin is mediated by integrin alpha 4 beta 1, J. Biol. Chem., 1992, 267, 1401914026.

25 M. S. Hahn, L. J. Taite, J. J. Moon, et al., Photolithographic patterning of polyethylene glycol hydrogels, Biomaterials, 2006, 27, 2519-2524.

26 Y. Liu, T. T. Yang Tan, S. Yuan, et al., Multifunctional P(PEGMA)-REDV conjugated titanium surfaces for improved endothelial cell selectivity and hemocompatibility, J. Mater. Chem. B, 2013, 1, 157-167.

27 H. Ceylan, A. B. Tekinay and M. O. Guler, Selective adhesion and growth of vascular endothelial cells on bioactive peptide nanofiber functionalized stainless steel surface, Biomaterials, 2011, 32, 8797-8805.

28 S. Yuan, G. Xiong, F. He, et al., Multifunctional REDVconjugated zwitterionic polycarboxybetainepolycaprolactone hybrid surfaces for enhanced 
antibacterial activity, anti-thrombogenicity and endothelial cell proliferation, J. Mater. Chem. B, 2015, 3, 8088-8101.

29 J. A. Hubbell, S. P. Massia, N. P. Desai, et al., Endothelial Cell-Selective Materials for Tissue Engineering in the Vascular Graft Via a New Receptor, Nat. Biotechnol., 1991, 9, 568-572.

30 W. Seeto, Y. Tian and E. Lipke, Peptide-grafted poly (ethylene glycol) hydrogels support dynamic adhesion of endothelial progenitor cells, Acta Biomater., 2013, 9, 8279-8289.

31 B. D. Plouffe, D. N. Njoka, J. Harris, et al., Peptide-Mediated Selective Adhesion of Smooth Muscle and Endothelial Cells in Microfluidic Shear Flow, Langmuir, 2007, 23, 5050-5055.

$32 \mathrm{H}$. Chen, X. Li, Y. Zhao, et al., Construction of a multifunctional coating consisting of phospholipids and endothelial progenitor cell-specific peptides on titanium substrates, Appl. Surf. Sci., 2015, 347, 169-177.

33 R. G. Thakar, F. Ho, N. F. Huang, et al., Regulation of vascular smooth muscle cells by micropatterning, Biochem. Biophys. Res. Commun., 2003, 307, 883-890.

34 Y. Yao, K. Fukazawa, N. Huang, et al., Effects of 3,4dihydrophenyl groups in water-soluble phospholipid polymer on stable surface modification of titanium alloy, Colloids Surf., B, 2011, 88, 215-220.

35 M. Ak, A. Cirpan, F. Yllmaz, et al., Synthesis and characterization of a bifunctional amido-thiophene monomer and its copolymer with thiophene and electrochemical properties, Eur. Polym. J., 2005, 41, 967-973.

36 X. Tan, J. Zhan, Y. Zhu, et al., Improvement of Uveal and Capsular Biocompatibility of Hydrophobic Acrylic Intraocular Lens by Surface Grafting with 2Methacryloyloxyethyl Phosphorylcholine-Methacrylic Acid Copolymer, Sci. Rep., 2017, 7, 40462.

37 Z. Yang, Q. Tu, J. Wang, et al., The role of heparin binding surfaces in the direction of endothelial and smooth muscle cell fate and re-endothelialization, Biomaterials, 2012, 33, 6615-6625.

38 J. Y. Shen, M. B. Chan-Park, B. He, et al., Three-dimensional microchannels in biodegradable polymeric films for control orientation and phenotype of vascular smooth muscle cells, Tissue Eng., 2006, 12, 2229-2240.

39 T. Konno, H. Hasuda, K. Ishihara, et al., Photoimmobilization of a phospholipid polymer for surface modification, Biomaterials, 2005, 26, 1381-1388.

40 R. Iwata, P. Suk-In, V. P. Hoven, et al., Control of nanobiointerfaces generated from well-defined biomimetic polymer brushes for protein and cell manipulations, Biomacromolecules, 2004, 5, 2308-2314.

41 Y. Ji, Y. Wei, X. Liu, et al., Zwitterionic polycarboxybetaine coating functionalized with REDV peptide to improve selectivity for endothelial cells, J. Biomed. Mater. Res., Part A, 2012, 100A, 1387-1397.

42 T. Konno. Cytocompatible Phospholipid Polymers for Noninvasive Nanodevices, Intracellular Delivery II, Springer, 2014. pp. 255-64.

43 P. Liu, Q. Chen, L. Li, et al., Anti-biofouling ability and cytocompatibility of the zwitterionic brushes-modified cellulose membrane, J. Mater. Chem. B, 2014, 2, 7222-7231.
44 M. Arnaout, B. Mahalingam and J.-P. Xiong, Integrin structure, allostery, and bidirectional signaling, Annu. Rev. Cell Dev. Biol., 2005, 21, 381-410.

45 S. L. Bellis, Advantages of RGD peptides for directing cell association with biomaterials, Biomaterials, 2011, 32, 42054210.

46 H. Shin, S. Jo and A. G. Mikos, Biomimetic materials for tissue engineering, Biomaterials, 2003, 24, 4353-4364.

47 B. A. Butruk-Raszeja, M. S. Dresler, A. Kuźmińska, et al., Endothelialization of polyurethanes: Surface silanization and immobilization of REDV peptide, Colloids Surf., B, 2016, 144, 335-343.

48 J. A. Hubbell, S. P. Massia, N. P. Desai, et al., Endothelial Cell-Selective Materials for Tissue Engineering in the Vascular Graft Via a New Receptor, Nat. Biotechnol., 1991, 9, 568-572.

49 R. Luo, L. Tang, S. Zhong, et al., In vitro investigation of enhanced hemocompatibility and endothelial cell proliferation associated with quinone-rich polydopamine coating, ACS Appl. Mater. Interfaces, 2013, 5, 1704-1714.

50 Z. Yang, J. Wang, R. Luo, et al., The covalent immobilization of heparin to pulsed-plasma polymeric allylamine films on $316 \mathrm{~L}$ stainless steel and the resulting effects on hemocompatibility, Biomaterials, 2010, 31, 2072-2083.

51 J.-H. Jiang, L.-P. Zhu, X.-L. Li, et al., Surface modification of $\mathrm{PE}$ porous membranes based on the strong adhesion of polydopamine and covalent immobilization of heparin, $J$. Membr. Sci., 2010, 364, 194-202.

52 Y. Liu, W.-Z. Qiu, H.-C. Yang, et al., Polydopamine-assisted deposition of heparin for selective adsorption of lowdensity lipoprotein, RSC Adv., 2015, 5, 12922-12930.

53 L. Lu, Q. L. Li, M. F. Maitz, et al., Immobilization of the direct thrombin inhibitor-bivalirudin on $316 \mathrm{~L}$ stainless steel via polydopamine and the resulting effects on hemocompatibility in vitro, J. Biomed. Mater. Res., Part A, 2012, 100, 2421-2430.

54 J. Park, T. F. Brust, H. J. Lee, et al., Polydopamine-based simple and versatile surface modification of polymeric nano drug carriers, ACS Nano, 2014, 8, 3347-3356.

55 J. Zhou, Y.-S. Li and S. Chien, Shear stress-initiated signaling and its regulation of endothelial function, Arterioscler., Thromb., Vasc. Biol., 2014, 34, 2191-2198.

56 J.-J. Chiu and S. Chien, Effects of disturbed flow on vascular endothelium: pathophysiological basis and clinical perspectives, Physiol. Rev., 2011, 91, 327-387.

57 D. Lu and G. S. Kassab, Role of shear stress and stretch in vascular mechanobiology, J. R. Soc., Interface, 2011, 8, 1379-1385.

58 L. Stoner, M. L. Erickson, J. M. Young, et al., There's more to flow-mediated dilation than nitric oxide, J. Atheroscler. Thromb., 2012, 19, 589-600.

59 J. Li, K. Zhang, P. Yang, et al., Human vascular endothelial cell morphology and functional cytokine secretion influenced by different size of HA micro-pattern on titanium substrate, Colloids Surf., B, 2013, 110, 199-207. 
60 J. Lei, Y. Vodovotz, E. Tzeng, et al., Nitric oxide, a protective molecule in the cardiovascular system, Nitric Oxide, 2013, 35, 175-185.

61 W. Feng, S. Zhu, K. Ishihara, et al., Adsorption of fibrinogen and lysozyme on silicon grafted with poly (2methacryloyloxyethyl phosphorylcholine) via surfaceinitiated atom transfer radical polymerization, Langmuir, 2005, 21, 5980-5987.
62 W. Feng, J. L. Brash and S. Zhu, Non-biofouling materials prepared by atom transfer radical polymerization grafting of 2-methacryloloxyethyl phosphorylcholine: Separate effects of graft density and chain length on protein repulsion, Biomaterials, 2006, 27, 847-855.

63 W. Feng, J. Brash and S. Zhu, Atom-transfer radical grafting polymerization of 2-methacryloyloxyethyl phosphorylcholine from silicon wafer surfaces, J. Polym. Sci., Part A: Polym. Chem., 2004, 42, 2931-2942. 